\title{
L'œuvre et les phénomènes. Le Baudelaire de Benjamin
}

\section{Clemens-Carl Härle}

\section{(2) OpenEdition}

Journals

\section{Édition électronique}

URL : http://journals.openedition.org/rief/625

DOI : $10.4000 /$ rief.625

ISSN : 2240-7456

\section{Éditeur}

Seminario di filologia francese

\section{Référence électronique}

Clemens-Carl Härle, «L'œuvre et les phénomènes. Le Baudelaire de Benjamin », Revue italienne d'études françaises [En ligne], 4 | 2014, mis en ligne le 15 décembre 2014, consulté le 30 avril 2019. URL : http:// journals.openedition.org/rief/625; DOI : 10.4000/rief.625

Ce document a été généré automatiquement le 30 avril 2019

\section{(c) (i) (9)}

Les contenus de la RIEF sont mis à disposition selon les termes de la Licence Creative Commons Attribution - Pas d'Utilisation Commerciale - Pas de Modification 4.0 International. 


\title{
L'œuvre et les phénomènes. Le Baudelaire de Benjamin
}

\author{
Clemens-Carl Härle
}

\section{NOTE DE L'AUTEUR}

Conférence prononcée à l'occasion de la Journée d'études sur Walter Benjamin « Les outils de la critique », organisée par K. Genel et G. Raulet en avril 2014 à l'Université Paris-Sorbonne.

1 Le titre que j'ai voulu donner à cette contribution sur le Baudelaire de Benjamin - L'œuvre et les phénomènes - peut être compris de plusieurs manières. D'abord, ce titre souligne l'importance des liens entre l'œuvre littéraire de Baudelaire et son époque, comme l'indique expressément le sous-titre que Benjamin aurait voulu donner au livre sur Baudelaire: Ein Lyriker im Zeitalter des Hochkapitalismus (Un poète lyrique à l'apogée du (capitalisme) ${ }^{1}$. Souvent, notamment dans la section $\mathrm{N}$ du Passagen-Werk - dont le Baudelaire aurait dû fournir un «modèle en miniature $»^{2}$ - ou dans les Thèses sur le concept d'histoire, Benjamin a essayé d'élucider les présupposés épistémologiques de ces liens. Ce titre se réfère aussi au fait que ce livre n'existe pas, que l'œuvre manque ou qu'elle est restée inaccomplie. Il indique alors le décalage entre l'œuvre absente et les traces plus ou moins dispersées, plus ou moins élaborées que son écriture partielle nous a léguées.

2 Mais le titre peut être compris en un autre sens. «Das Werk ist die Totenmaske seiner Konzeption » («L'œuvre est le masque mortuaire de sa conception »), écrit Benjamin dans Einbahnstraße $e^{3}$. C'est la dernière des treize thèses sur la "technique de l'écrivain", publiées pour la première fois en octobre 1925 dans Die literarische Welt. Toute œuvre, semble suggérer l'aphorisme de Benjamin, qu'elle soit accomplie ou inaccomplie, est un phénomène. Elle est le phénomène visible, la trace, plus ou moins lisible, de son idée, en quelque sorte sa "facies hippocratica ». Selon la théorie de l'allégorie élaborée dans l' Origine du drame baroque allemand, l'œuvre serait le «paysage primitif pétrifié » de son 
idée et toute réalisation d'une idée, l'écriture en première instance, serait une mise à mort de cette idée même ${ }^{4}$. Dans le cas du Baudelaire, dans le cas du livre absent, on ne saurait même pas parler de masque mortuaire. Car un masque mortuaire s'offre encore sous l'aspect d'un visage ou d'une forme, d'un ensemble reconnaissable de traits. L'absence du livre n'est, en revanche, que la pure dispersion des phénomènes ou des fragments, un chantier ou une ruine avant la lettre, la ruine d'un objet qui n'aura jamais existé et dont nous cherchons à reconstruire le tracé, la figure ou l'idée.

Mais le syntagme «L'œuvre et les phénomènes » peut être encore décliné d'une autre manière. Le mot phénomènes ne se réfère pas uniquement aux phénomènes extra- ou nonlittéraires évoquées par Benjamin, à savoir les phénomènes historiques ou contingents au sens propre du terme. Par phénomènes il faut aussi entendre les phénomènes infralittéraires, tout ce qui apparaît et existe à l'intérieur d'une œuvre en tant que contenu, tout ce à quoi Baudelaire semble se référer quand il déclare dans Le cygne « Tout pour moi devient allégorie », phrase que Benjamin cite en exergue à l'Exposé de 1934.

C'est de ce dernier aspect que je voudrais parler d'abord, à savoir de la fonction de l'allégorie comme trope littéraire qui fait la singularité de l'œuvre de Baudelaire. Car en dépit de ce que Gilles Deleuze aurait pu appeler le « cri » de Baudelaire - « Tout pour moi devient allégorie »-, le statut de ce que Benjamin définit comme « la vision allégorique » de Baudelaire ou plus précisément «la forme allégorique de l'intuition », en allemand allegorische Anschauungsform, est loin d'être claire ou univoque. Ce serait une erreur, telle est la position de Benjamin, de comprendre l'allégorie baudelairienne à partir d'une disposition subjective ou affective. Bien au contraire : «L'introduction de l'allégorie doit être déduite de la situation de l'art tel qu'il est déterminé par le développement technique ; et c'est seulement sous le signe de celui-ci qu'on peut concevoir la disposition mélancolique de cette poésie » (B 662). C'est la raison pour laquelle, dans la première partie du livre, intitulée selon les documents dont nous disposons «Idée et images », la section consacrée à l'allégorie précède la section consacrée à la mélancolie. D’autre part, il faut admettre que si « la vision allégorique [...] avait été à l'origine d'un style au XVII siècle, [elle] ne l'était plus aux XIX ${ }^{\mathrm{e}}{ }^{5}$. En tant qu'allégoriste, donc, «Baudelaire était un cas isolé ; son isolement était, d'un certain point de vue, celui d'un retardataire » (B 667). Ni disposition subjective au sens psychologique du terme, ni expression d'un style comme dans le baroque, la situation de «l'intention allégorique ( (B 645) de Baudelaire semble plutôt aporétique. Dans une note de Zentralpark, Benjamin expose clairement la question dont la réponse constitue un des enjeux majeurs du livre: «Comment se fait-il qu'un mode de comportement qui est de bout en bout, du moins en apparence, aussi 'inactuel' mot de Nietzsche repris par Benjamin - que celui de l'allégoriste occupe dans l'œuvre poétique du siècle une des toutes premières places? » (B 654).

5 Pour essayer de donner une réponse à cette question, il faut d'abord comparer le statut de l'allégorie dans le Baudelaire avec la théorie de l'allégorie dans le Trauerspielbuch. Plusieurs fragments suggèrent de procéder ainsi. Dans les deux cas, pourrait-on dire, le rapport entre le signe et le sens s'organise autour d'une coupure pour ainsi dire transcendantale : l'allégorie est un signe dont le sens est toujours indirect ou caché, c'est-à-dire non déductible par analogie ou par anagogie. Dans l'allégorie baroque, c'est la mort qui opère cette coupure, cette séparation radicale entre le signifiant et le signifié, et il n'y a de 
signification que dans la mesure où quelque chose - un corps, une nature - est livré à la mort: "Autant de sens, autant d'emprise de la mort, parce que la mort inscrit au plus profond la ligne de démarcation brisée qui sépare la physis et le sens » (Soviel Bedeutung, soviel Todverfallenheit, weil am tiefsten der Tod die zackige Demarkationslinie zwischen Physis und Bedeutung eingräbt)». Ainsi le modus significandi de l'allégorie est toujours thesei codifié ou conventionnel - et jamais physei, et la physis ou la nature est précisément ce qui apparaît ou devient expressif à la suite d'une violence: un organisme dé-taillé, martyrisé, une totalité démembrée, un flux ou un mouvement figé et paralysé, privé de finalité interne, trace matérielle de l'irruption d'un dehors. Dans le cas de l'allégorie baroque, ce dehors, le prétexte du texte allégorique, s'annonce sous la forme d'une théologie: «la signification et la mort sont autant les produits du développement de l'histoire que des germes, imbriqués l'un dans l'autre, dans l'état de péché de la créature exclue de la grâce » (0 179).

Chez Baudelaire, cette codification théologique si essentielle à l'allégorie baroque est comme pulvérisée. L'allégorie ne s'annonce plus à travers des masques ou des emblèmes de la mort et de la finitude. "L'allégorie baroque ne voit le cadavre que de l'extérieur, Baudelaire de l'intérieur » observe Benjamin, d'ailleurs sans référence explicite à Une charogne (B 662). «La figure clé de l'allégorie d'alors est le cadavre. La figure clé de l'allégorie tardive est la 'remémoration' (Andenken)» (B 666). Chez Baudelaire l'allégorie est pour ainsi dire mise à nu, simple forme du temps dont la finitude n'est qu'une figure parmi d'autres, à côté d'autres figures, modalités ou postures immédiatement temporelles comme la répétition, le toujours-le-même, la nouveauté, la remémoration, La vie antérieure. "Le moi de Baudelaire est un étrange sectionnement des temps où seuls de rares jours notables apparaissent ", écrit Proust dans son essai sur Baudelaire, cité par Benjamin (B 45). «Et le temps m'engloutit minute par minute, / Comme la neige immense un corps pris de roideur» (Le goût du néant). L'allégorie est la forme de l'intériorité, le sens interne: «Au dix-neuvième siècle, l'allégorie a abandonné le monde environnant pour s'établir dans le monde intérieur. La relique vient du cadavre, la remémoration ( Andenken) de l'expérience morte que l'on avait (abgestorbene Erfahrung)» (B 658). Enfin, la mort même doit être soumise au temps : «Il n'existe pour les hommes tels qu'ils sont aujourd'hui qu'une nouveauté radicale - et c'est toujours la même : la mort » (B 647)6.

7 Si l'allégorie baudelairienne est « l'antidote du mythe » (B 655), c'est parce qu'elle porte au contraire de l'allégorie baroque - «les traces de la colère rentrée (Ingrimm) qui était nécessaire pour pénétrer par effraction dans ce monde et réduire à l'état de ruine ces œuvres harmonieuses » (B 649). Ce sont les démolitions et les changements de l'aspect de la ville - " palais neufs, échafaudages, blocs, / Vieux faubourgs » - qui font dire au moi lyrique : « Tout pour moi devient allégorie » et qui font apparaitre « l'antiquité au travers de la modernité » (B 67). "Cette ville prise dans un mouvement incessant se fige. Elle devient cassante comme le verre, mais aussi transparente comme lui - en laissant voir ce qu'elle signifie [...] La stature de Paris est fragile; elle est cernée de symboles de décrépitude: créatures vivantes - la négresse et le cygne, et personnages historiques - Andromaque, 'veuve d'Hector, hélas! et femme d'Hélénus'. Leur trait commun est la déploration de ce qui a été et l'absence d'espoir en ce qui sera » (B 776)7 .

Une des conséquences majeures de l'irruption du temps - de l'irruption du dehors sous la simple forme du temps - est la transformation de l'objet en image. "Idée et image », je l'ai déjà dit, aurait dû être le titre de la première partie du livre. Benjamin cite Joubert : « Les poëtes sont plus inspirés par les images que par la présence même des objets », mais 
surtout il ajoute que c'est le temps qui provoque cette déréalisation, car « ce dont on sait qu'on ne l'aura bientôt plus sous les yeux devient image » (B 780). C'est la raison pour laquelle Benjamin souligne que chez Baudelaire «l'intérêt initial pour l'allégorie n'est pas d'ordre verbal, mais d'ordre visuel » (B 663). «L'image, ma grande, ma primitive passion » fait écho à "Tout pour moi devient allégorie $»^{8}$. Le renvoi au miroir - «ce Simoïs menteur» - au début du Cygne que Benjamin ne mentionne pas mais dans lequel se réfléchit le deuil d'Andromaque et dont l'image " féconde soudain ma mémoire fertile " confirme le rapport intime entre l'allégorie, l'irruption du temps, l'absence d'objet, l'image et le souvenir. C'est l'image - et non l'expérience sensible - qui est mise « à tout moment [...] au service de la pensée » (B 648).

9 Comme l'indiquent les schèmes préparatoires et la lettre à Horkheimer d'avril 1938, le motif des correspondances aurait dû être traité dans la première partie du livre, qui avait "pour tâche d'élucider le paradoxe» de la doctrine artistique de Baudelaire, «la contradiction entre la théorie des correspondances naturelles et le refus de la nature» sanctionné par l'allégorie $(B 67)^{9}$. On peut risquer l'hypothèse selon laquelle au moment où il esquissait ce programme Benjamin ne disposait pas des moyens conceptuels suffisants pour résoudre cette contradiction. C'est peut-être une des raisons pour lesquelles il commença, en août 1938, la rédaction du livre en écrivant d'abord la deuxième partie, Le Paris du Second Empire chez Baudelaire. Ce n'est qu'au printemps de l'année suivante, lors de la réécriture du chapitre sur le flâneur, que le recours à la théorie de l'inconscient élaborée par Freud dans Au-delà du principe de plaisir permettra d'affronter la «contradiction". L'esthétique des correspondances, à première vue incompatible avec la logique de l'allégorie, peut lui être associée, si l'on accepte, suivant l'hypothèse freudienne, que le temps est un mécanisme de défense, que l'inconscient ne connaît pas le temps et que les traces mnésiques s'inscrivent dans l'inconscient sans passer par la conscience/perception occupée à parer l'attaque des impressions. Une sorte de passé en soi et immémorial fonctionne comme condition temporelle du jeu des correspondances baudelairiennes qui - c'est la rectification d'un préjugé partagé par la quasi-totalité des interprètes - ne sont pas «simultanées comme celles que cultiveront plus tard les symbolistes ». Dans les correspondances, le "passé murmure » et en elles se conservent les traces d'un présent qui n'a jamais eu lieu ; ainsi « l'expérience canonique » qu'on peut en avoir «trouve sa place dans une vie antérieure » (B 981) ${ }^{10}$. Si l'allégorie de Baudelaire exprime la forme du temps comme condition de toute signification, les correspondances représentent un cas limite de l'allégorèse. Elles ne sont pas un miroir où se réfracte un passé, devenu ainsi accessible à une mémoire présente, mais le lieu où s'énonce un passé absolu. Le Eingedenken, la «remémoration » ne peut présenter ce passé que comme relevant d'une " préhistoire ", comme quelque chose qui n'a jamais été vécu, c'est-à-dire comme un passé plus bergsonien que proustien, s'il est vrai, comme l'affirme Deleuze, que la mémoire involontaire doit être comprise comme une tentative d'accéder à l'en-soi du passé, de le sauver pour nous ${ }^{11}$. Benjamin toutefois semble identifier les deux, le passé de Bergson et celui de Proust, en les opposant, sous le nom d'Erfahrung, à l' Erlebnis, l'expérience vécue entièrement engagée dans l'effort de parer le choc produit par les impressions de la grande ville. 

allégorique évoquée dans les papiers du Baudelaire. Benjamin soutient que l'époque du Hochkapitalismus - du capitalisme à son apogée - doit être comprise elle-même à partir d'une modalité de l'allégorie. Dans sa conception générale - mais avec des exceptions, dont font partie les renvois aux événements politiques et surtout à la figure de Blanqui -, le Hochkapitalismus ne peut pas être conçu comme un ensemble de phénomènes qui se montreraient à partir d'eux-mêmes et seraient plus ou moins lisibles, mais comme une phénoménalité dégradée et désarticulée sous l'effet de la forme-valeur, c'est-à-dire de la marchandise. De l'analyse marxienne de la marchandise Benjamin retient surtout son caractère de fétiche : s'il est vrai - comme écrit Marx - que la valeur «fait de chaque produit du travail un hiéroglyphe " (B 448), alors on peut entrevoir dans la marchandise une modalité non-littéraire - « prosaïque » - de l'allégorie.

Affirmer que la marchandise est une forme de l'allégorie n'est pas quelque chose d'évident. On se souvient que Marx rejetait les tentatives de réduire le rapport social à un rapport de signification ou de le comprendre dans l'horizon d'un rapport de signification. L'interprétation des «caractères matériels que revêtent les déterminations sociales du travail sur la base d'un mode particulier de production » en tant que "simples signes » risque, selon Marx, de transformer ces « caractères matériels » en un " produit arbitraire de la pensée des hommes (willkürliches Reflexionsprodukt) $»^{12}$. En d'autres termes, pour Marx le signe semble être un fait purement mental qui suscite des "subtilités métaphysiques » et des "arguties théologiques ». Benjamin, en revanche, insiste sur le fait que le signe n'est pas une entité imaginaire, mais bien réelle : «Toute marchandise est un signe » (B 447). Et c'est précisément parce qu'il donne un statut ontologique à l'inversion fétichiste, qu'il peut affirmer le caractère allégorique de la marchandise. L'affichage de son prix en est pour ainsi dire l'emblème, cette " objectivité spectrale » ( $B$ 448) qui témoigne de l'arbitraire de la signification, «car il n'y a pas de médiation naturelle » entre les deux, entre la marchandise et son prix (B 471).

Si une chose quelconque n'a pas, en tant que marchandise, d'autre signification que son prix, il en résulte un "avilissement singulier des choses par leur signification ", à savoir "par leur prix comme marchandise» $\left(B\right.$ 847) ${ }^{13}$. Cet avilissement opéré par la forme allégorique de la marchandise est encore plus violent que la mise en suspens du monde matériel (Dingwelt) opérée par l'allégorie littéraire. «La dépréciation du monde matériel dans l'allégorie est, à l'intérieur du monde matériel lui-même, l'objet d'une surenchère de la part de la marchandise » (B 639). Parfois Benjamin évoque un remplacement du trope littéraire par la marchandise: «La marchandise a pris la place du mode de vision ( Anschauungsform) allégorique " (B 663). Mais le rapport entre les deux modalités de l'allégorie est plus complexe qu'il ne semble à première vue, et il est donc irréductible à une simple analogie. Si le trope détruit le semblant - l'allégorie est un «antidote du mythe »-, la marchandise le renforce. « La destruction de l'organique, du vivant » qui est à l'œuvre dans "l'intention allégorique » opère un effacement ou "dissipation de l'apparence (Auslöschung des Scheins) » (B 648), la marchandise transforme le monde phénoménal en fantasmagorie. Comme on l'a vu dans le cas du Cygne, la mise en suspens du monde phénoménal sous l'impact de la forme allégorique du temps produit des images virtuelles qui se substituent à la simple présence sensible. L'allégorie est antimythologique précisément parce qu'elle détruit le semblant de l'organique. Mais le vidage de la consistance matérielle du monde dû à la transformation du phénomène en hiéroglyphe social ou simple signe de valeur va de pair avec l'instauration des 
fantasmagories, c'est-à-dire avec la transfiguration imaginaire d'un monde devenu obtus et opaque ${ }^{14}$. La fantasmagorie est beaucoup plus qu'une simple «transposition idéologique » produite par la conscience, elle est une «illumination (Verklärung) » qui a lieu «dans l'immédiateté de la présence sensible ${ }^{15}$. L'analyse des Sept vieillards, la deuxième des poésies dédiées à Victor Hugo, suggère par exemple que l'effet fantasmagorique de la flânerie résulte de la subsomption d'un phénomène ou événement singulier sous la catégorie de l'égalité ou de l'équivalence. "L'individu qui est ainsi présenté dans sa multiplication comme toujours le même témoigne de l'angoisse du citadin à ne plus pouvoir [...] rompre le cercle magique du type " (B 847). La fantasmagorie opère à la fois au niveau de la perception, de l'affect, et du mental, en instaurant un circuit d'images virtuelles indiscernables de l'image actuelle. Le même mécanisme infernal, basé sur la multiplication des images virtuelles identiques de l'instant actuel, semble être à l'œuvre dans les fantasmagories du temps dont la description était réservée à la troisième partie du livre : l'éternel retour de Nietzsche, les spéculations cosmiques de Blanqui et les monstres produits par le spleen.

Je voudrais à présent aborder une dernière question qui me paraît essentielle pour la compréhension du Baudelaire, à savoir la manière dont Benjamin conçoit et construit le rapport entre le texte littéraire et l'ensemble de l'époque dont il fait partie.

Par rapport aux autres écrits de Benjamin sur la littérature ou la poésie, le Baudelaire est sûrement une exception. Le livre vise l'unité de l'œuvre de l'auteur, sans pour autant tenir compte de l'unité des œuvres singulières dont l'ensemble de l'œuvre se compose. «La réalité suprême de l'art, c'est l'œuvre isolée, fermée » affirmait Benjamin dans la Préface épistémo-critique du Drame baroque (O 54). La lecture de deux poèmes d'Hölderlin, Dichtermut et Blödigkeit (1917) et des Affinités électives de Goethe (1922) obéit à ce critère, tandis que les analyses consacrées au drame baroque et l'essai sur Kafka ne le respectent pas, même si c'est pour des raisons différentes. Dans le premier cas, on recherche la singularité d'un genre littéraire, dans le deuxième la spécificité de l'œuvre d'un auteur. Dans les deux cas, Benjamin met en question l'unité de sens du texte singulier - comme d'ailleurs dans le Baudelaire, où À une passante est le seul poème reproduit entièrement dans les deux rédactions partielles - et procède en choisissant des motifs spécifiques et transversaux qui démembrent les textes singuliers. «Accentuant l'aspect fragmentaire de l'œuvre d'art " (B 667), cette manière de procéder répète le morcellement allégorique. Dans le cas du Trauerspielbuch, Benjamin la justifie en affirmant que «l'âge baroque est plutôt celui d'un vouloir artistique acharné que d'un véritable exercice de l'art", affirmation qui reprend le concept de Riegl qui avait « découvert ce terme - Kunstwollen en étudiant précisément la dernière phase de l'art romain » (0 54). Mais ce qui constitue la singularité du Baudelaire est non seulement la tentative de saisir l'unité d'une œuvre littéraire, mais de présenter l'œuvre de Baudelaire come un tout où se dessine en creux la physionomie - la facies - d'une époque entière. Benjamin suggère ainsi que l'œuvre de Baudelaire resterait incompréhensible sans ce recours à un ordre qui, d'une certaine façon, le précède. Dans une note de Zentralpark, il écrit que «ce travail doit fournir la projection historique des expériences qui sont à la base des Fleurs du mal» (B 651). Comment peut-on comprendre cette déclaration dont la construction du livre doit fournir la preuve? 

ontologique entre l'œuvre d'art et les phénomènes historiques, qu'Aristote avait établie dans le chapitre 9 de la Poétique. "La poésie est plus philosophique et plus noble que la chronique ", parce qu'elle "traite plutôt du général (ta katholou) », tandis que l'histoire s'occupe du particulier (kath'ekhaston). La poésie montre ce qui «pourrait avoir lieu dans l'ordre du vraisemblable ou du nécessaire», l'histoire présente «ce qui a lieu réellement » et qui, étant soumis à la contingence, n'a ni « vraisemblance ni nécessité " (1451 b 4 sq. $)^{16}$. Dans le cas du Baudelaire, il ne s'agit pas d'un renversement pur et simple, parce que pour Benjamin il ne s'agit pas tant de subordonner la critique littéraire au discours de l'historien, mais de fournir 'seulement' la «projection historique des expériences qui sont à la base des Fleurs du mal». On pourrait peut-être comprendre l'expression «projection historique » de la façon suivante : l'œuvre littéraire est la ratio cognoscendi de l'histoire des événements, et l'histoire est la ratio essendi de l'œuvre. Il revient donc à la critique d'établir les règles de la "projection" pour identifier les «expériences » qui sont à la base des Fleurs du mal. C'est en ce sens que dans l'Exposé français du printemps 1939, Benjamin peut écrire que « la clé de la forme allégorique chez Baudelaire est solidaire de la signification spécifique que prend la marchandise du fait de son prix» (B 847). "Solidarité", et non pas coïncidence ou identité. La « projection historique », donc, est loin d'abolir l'hétérogénéité de principe entre la forme poétique de l'allégorie - le trope - et la forme pour ainsi dire prosaïque de l'allégorie qui est à l'œuvre dans la marchandise, c'est-à-dire entre les images et les fantasmagories. Le problème que Benjamin doit affronter est donc double : il ne doit pas seulement fixer les règles de la " projection ", mais aussi envisager ce qu'on pourrait nommer une « synthèse de l'hétérogène ", une synthèse entre l'expérience historique et son expression, son articulation - ou désarticulation - littéraire.

C'est en vue d'une telle synthèse, me semble-t-il, que dans la dix-septième thèse sur le concept d'histoire - où l'on trouve énoncé pour ainsi dire le principe de construction du Baudelaire - Benjamin reprend le motif monadologique. Ce motif avait été déjà évoqué dans la Préface épistémo-critique du Trauerspielbuch, bien que sous un angle un peu différent, à savoir par rapport à l'idée : «L'idée est monade - ce qui signifie en résumé : toute idée renferme l'image du monde » (O 46). Ainsi protégé dans l'immanence de l'idée, «le devenir des phénomènes [est établi, festgestellt] dans leur être» ( 045$)$. C'est précisément grâce à la forme monadologique de l'idée de l'origine que le devenir et la dispersion des phénomènes acquièrent un sens et un ordre en tant que "pré- et posthistoire ». Si «l'origine est un tourbillon dans le fleuve du devenir », un tourbillon qui «entraîne dans son rythme la matière de ce qui est en train de naître » ( 0 43), «l'histoire philosophique, considérée comme science de l'origine, est la forme qui fait procéder des extrêmes éloignés, des excès apparents de l'évolution, la configuration de l'idée, c'est-à-dire la totalité où de telles oppositions peuvent coexister d'une manière qui fasse sens » (0 45). La monade est l'opérateur d'une totalisation et d'une abréviation. Dans l'idée, «la représentation des phénomènes repose, préétablie, en tant qu'elle est leur interprétation objective » $(046)$, et l'histoire n'y «apparait plus que comme frange colorée d'une simultanéité cristalline » (0 35).

Par rapport à la Préface du Drame baroque, le motif monadologique apparaît profondément modifié dans les Thèses. Dans la Préface, la doctrine des idées avait été introduite en opposition aux théories nominalistes et aux classifications simplement conceptuelles des formes de l'art. Dans la dix-septième thèse, les adversaires sont 
l'historicisme et le projet d'une histoire universelle auxquels la «cristallisation monadologique » s'oppose comme " principe constructif ». Mais ce qui est plus important encore que le contexte polémique, c'est le changement dans la manière dont Benjamin conçoit le rapport entre la pensée et le temps. Si dans la thèse il n'est plus question de l'idée comme forme de la présentation des phénomènes, c'est parce qu'à sa place s'établit un nouveau lien entre la pensée et le temps, qui s'exprime dans la formule Jetzt der Erkennbarkeit. Dans la seizième thèse, Benjamin écrit : «L'historien matérialiste ne saurait renoncer au concept d'un présent qui n'est point passage, mais arrêt et blocage du temps. Car un tel concept définit justement le présent dans lequel, pour sa part, il écrit l'histoire. L'historicisme compose l'image 'éternelle' du passé, le matérialisme historique dépeint l'expérience unique de la rencontre avec ce passé $»^{17}$. Lier la pensée au temps - parler d'une « rencontre » avec le passé qui est l'objet de la connaissance historique, et parler d'un présent « qui n'est point passage, mais arrêt et blocage du temps » - cela signifie concevoir un présent qui n'est ni un instant fugitif dans la suite des instants, un présent encastré entre le passé et futur, ni a fortiori une instance extra- ou a-temporelle.

On pourrait préciser l'enjeu conceptuel de cet entrelacement entre la pensée et le temps, en faisant recours à la distinction entre histoire et mémoire, proposée par Péguy dans Clio - «L'histoire est essentiellement longitudinale, la mémoire est verticale. L'histoire consiste essentiellement à passer au long de l'événement. La mémoire consiste essentiellement, étant dedans l'événement, avant tout à n'en pas sortir, à y rester, et à le remonter en dedans ${ }^{18}$. L'histoire universelle est longitudinale, elle "procède par addition : elle mobilise la masse des faits pour remplir le temps homogène et vide ». En revanche, la mémoire, c'est-à-dire le geste de s'enfoncer dans l'événement même, implique un blocage ou un arrêt - Stillstellung - de la pensée conçue comme flux ou comme mouvement. La phrase décisive dans la dix-septième thèse est (dans la traduction de Benjamin lui-même): "L'acte de penser - je dirais presque: la spontanéité de la pensée - ne se fonde pas seulement sur le mouvement des pensées, mais aussi sur leur blocage. Supposons soudainement bloqué le mouvement de la pensée - il se produira alors dans une constellation surchargée de tensions une sorte de choc en retour; une secousse qui vaudra à l'image, à la constellation qui la subira, de s'organiser à l'improviste, de se constituer en monade $»^{19}$. De la Préface du Trauerspielbuch, Benjamin retient donc ce qu'il appelle la "tendance de toute conceptualisation philosophique ", c'est-à-dire "fixer - feststellen - le devenir des phénomènes dans leur être ", ce qui implique une Auszehrung - consommation ou épuisement - de l'histoire ou du devenir ( $O$ 45). Mais si la pensée est elle-même exposée au temps et cherche, comme la mémoire chez Péguy, à s'enfoncer dans l'événement - ce qui n'était pas le cas dans la Préface -, elle ne peut plus s'appuyer sur l'idée - ou sur l'idée d'origine - pour effectuer l'arrêt de son propre mouvement. Bien au contraire, plongée dans une «constellation surchargée de tensions ", la pensée doit s'imposer elle-même un arrêt, doit pour ainsi dire suspendre spontanément sa propre spontanéité. C'est cette auto-affection de la pensée qui produit, grâce à la force qu'elle exerce sur elle-même, "une sorte de choc en retour » dans le milieu où elle est plongée. Ce choc transforme la constellation en monade. Il prend donc la place qui était réservée au tourbillon dans la logique de l'idée d'origine qui est celle de l'idée et fonctionne pour ainsi dire comme le germe de la cristallisation monadique.

Par rapport au Trauerspielbuch et a fortiori par rapport à Leibniz, le concept de monade a considérablement changé de statut. Dans le contexte des Thèses qui vaut tel quel pour le Baudelaire, la monade n'est plus simplement une forme ontologique, préexistante, mais 
l'effet d'un "choc en retour» qui transforme la succession temporelle dans une simultanéité ou une co-présence qui ressemble à ce que Deleuze appelle une imagetemps. Le choc que la pensée communique à son milieu n'est d'ailleurs pas tout à fait étranger à la violence que l'allégorie exerce sur le corps du signifiant. Mais à la différence de l'allégorie dont la structure implique une antériorité temporelle, dans l'arrêt monadologique de la pensée toute antériorité est effacée à la faveur d'une "simultanéité cristalline ».

L'effet du choc provoqué par l'auto-affection de la pensée est double. D'un côté, il fait " éclater la continuité historique ", la succession des événements et des figures infratemporelles soumises à la forme homogène du temps. De l'autre, il donne lieu à une totalisation immanente des phénomènes historiques dispersés qui se substitue à la totalisation en pré- et post-histoire, dont il était question dans la Préface. Dans ce circuit entre la destruction de la forme du temps et la restitution du phénomène dans l'imagecristal se produit ce que j'ai nommé "synthèse de l'hétérogène ", à savoir la disjonction inclusive entre l'événement littéraire et l'événement mondain ou historique qui deviennent ainsi indiscernables, sans pour autant être privés de leur différence de structure. Pour le Baudelaire, en effet, vaut exactement ce que Benjamin affirme à la fin de la dix-septième thèse à propos du modus operandi de l'historien matérialiste: «il fait éclater la continuité historique pour en dégager une époque donnée; il ira faire éclater pareillement la continuité d'une époque pour dégager une vie individuelle; enfin il ira faire éclater cette vie individuelle pour en dégager un fait ou une œuvre donnés ». Mais ce faisant, c'est-à-dire à travers ce mouvement de destruction progressive et descendante (du plus grand au plus petit) il réussira, écrit-il, à faire apparaître, dans un mouvement d'emboîtement contraire (du plus petit au plus grand), «à faire voir comment la vie entière d'un individu tient dans une de ses œuvres, un de ses faits; comment dans cette vie tient une époque entière ; et comment dans une époque tient l'ensemble de l'histoire humaine ». Bien que dans la version allemande des termes comme aufbewahren et aufheben puissent suggérer une certaine affinité avec la dialectique hégélienne, l'immanence du singulier dans l'universel - et plus encore de l'universel dans le singulier - ne se produit pas à travers le mouvement spéculatif du concept, mais à travers la cristallisation ou l'image monadologique du phénomène, donc non pas à un niveau ontologique, mais pour ainsi dire onto-chronique.

21 C'est ainsi, me semble-t-il, qu'il faut lire la phrase assez énigmatique avec laquelle se termine la thèse : «Les fruits nourrissants de l'arbre de la connaissance sont donc ceux qui portent enfermés dans leur pulpe, telle une semence précieuse mais dépourvue de goût, le Temps historique $»^{20}$. En s'enfonçant dans le phénomène ou dans l'événement, la connaissance historique produit une image virtuelle du phénomène ou de l'événement qui permet que le passé soit conservé dans une figure paradoxalement immobile et temporelle. En évoquant une "simultanéité cristalline» dans laquelle "l'histoire n'apparaît plus que comme [une] frange colorée ", la Préface du Drame baroque exprimait l'énigme de ce temps immobilisé à travers une métaphore optique. Dans la thèse, en revanche, on assiste à une métaphore organique-naturelle voire phallique dont les connotations théologiques sont beaucoup moins fortes dans la version allemande. À la présence subtile de la couleur se substitue une anesthésie générale, une privation de l'affection sensible, compensée par la promesse d'un «fruit » sans goût mais autrement nourrissant. Un fruit, nourrissant mais sans goût, qu'aucune consommation ne peut détruire, parce qu'il contient en son intérieur un temps indifférent au temps qui passe, un 
temps en soi, indifférent au temps pour nous. Est-ce un paradoxe, si Benjamin conçoit le travail de l'historien marxiste presque de la façon dont il a décrit le travail d'Hérodote dans l'article de 1936 sur le narrateur? À propos de la légende du roi égyptien Psammenit, qui, vaincu, reste impassible quand il reconnaît dans le défilé des prisonniers sa fille devenue servante et son fils qui attend l'exécution, mais qui est profondément ému quand il voit un vieux domestique appauvri, Benjamin avait écrit : « Elle ressemble à ces grains de blé qui, malgré un séjour de tant de siècles dans les chambres des Pyramides, conservent aujourd'hui encore toute leur puissance de germination $»^{21}$.

\section{NOTES}

1. W. Benjamin, Baudelaire, édition établie par G. Agamben, B. Chitussi et C.-C. Härle, Paris, La fabrique, 2013 (indiqué dorénavant par B).

2. W. Benjamin, Lettre à M. Horkheimer du 16.4.1938, in Gesammelte Briefe, VI, , Frankfurt/Main, Suhrkamp, 2000, p. 64.

3. W. Benjamin, Sens unique, Paris, Les lettres nouvelles, 1978, p. 170.

4. W. Benjamin, Origine du drame baroque allemand, Paris, Flammarion, 1985, p. 178 (dorénavant indiqué par 0 ).

5. Cf. aussi : « La marchandise n'avait pas encore marqué aussi profondément de son stigmate - la prolétarisation de ceux qui produisent - le processus de production. C'est pourquoi la vision ( Anschauung) allégorique était aux dix-septième siècle créatrice de style, alors qu'elle ne l'était plus au dix-neuvième. En tant qu'allégoriste, Baudelaire était isolé. Il a essayé de rapporter l'expérience et la marchandise à l'expérience allégorique » (B 463).

6. Paul de Man souligne à juste titre la structure temporelle de l'allégorie et la bifurcation entre le signe allégorique pour ainsi dire présent et le signe antécédent non-allégorique mais nécessairement impliqué dans l'allégorie : «It remains necessary, if there is to be allegory, that the allegorical sign refers to another sign that precedes it. The meaning constituted by the allegorical sign can than consist only in the repetition (in the Kierkegaardian sense of the term) of a previous sign with which it can never coincide, since it is of the essence of the the previous sign to be pure anteriority ", P. de Man, The Rhetoric of Temporality, in Blindness and Insight. Essays in the Rhetoric of Contemporary Criticism, University of Minnesota Press, Minneapolis 1983, p. 207.

7. Pour une lecture qui, partant du sonnet mallarméen "Le vierge, le vivace et le bel aujourd'hui", reconnaît dans le "cygne agité" de Baudelaire "le deuil de la journée révolutionnaire, toujours marquée par la défaite”, $c f$. J.-C. Milner, Mallarmé au tombeau, Lagrasse, Verdier, 1999.

8. Mais cette désactualisation qu'est l'image - et qui est le contraire d'une désaffection - ne résulte pas tant de la spontanéité de l'imagination que plutôt de la subordination de l'imagination productrice - «créatrice de formes arbitraires d'intuitions possibles » selon Kant sous la forme du temps, c'est-à-dire de la subordination paradoxale de l'imagination à elle-même. 9. Maintes notes de Zentralpark insistent sur la tension entre la forme allégorique et les correspondances sans l'élaborer conceptuellement. Pour en citer quelques-unes : « Le fondement déterminant de la production baudelairienne est la tension existant chez lui entre une sensibilité exacerbée et une contemplation portée à son plus haut point de concentration. Cette tension se retrouve, au niveau théorique, entre la doctrine des correspondances et la doctrine de l'allégorie. Baudelaire n'a jamais tenté d'établir le moindre lien entre ces deux axiomes qui lui tenaient le 
plus à cœur. Sa poésie naît de la conjugaison de ces deux tendances qui étaient en lui » (B 652). «Si le souvenir reçoit de l'imaginaire (Phantasie) les correspondances, c'est la pensée qui lui octroie l'allégorie. Le souvenir réunit imaginaire (Phantasie) et pensée " (B 647). D'autres notes répètent presque littéralement les observations contenues dans la lettre à Horkheimer (cf. B 103 et B 638). 10. Benjamin n'élimine pas absolument la poétique de l'art pour l'art de l'œuvre de Baudelaire : «Les théorèmes compliqués [...] à propos de l'art pour l'art [...] se résument plus ou moins en une phrase : la sensibilité est le vrai sujet de la poésie. La sensibilité est, par nature, souffrante. Si elle connaît sa concrétisation suprême, sa destinée la plus accomplie, dans l'érotisme, elle trouve son accomplissement absolu, qui coïncide avec sa sublimation, dans la passion. La poétique de l'art pour l'art est passée sans coup férir dans la passion poétique des Fleurs du mal » (B 644); cf. aussi la note J 79,6 du Passagen-Werk. Dans la première partie du Baudelaire, la section «tempérament sensible " précède la section " passion esthétique », suivie par « allégorie » et " mélancolie ».

11. Cf. G. Deleuze, Différence et répétition, Paris, PUF, 1968, p. 115.

12. K. Marx, Das Kapital, Berlin (Ost), Dietz, 1981, p. 105 ; cf. aussi B 447.

13. Cf. aussi la note $\mathrm{J} 80,2 / \mathrm{J} 80 \mathrm{a}, 1$ du Passagen-Werk.

14. Cf. aussi la note J 67, 2 du Passagen-Werk: "La 'valeur' comme miroir ardent naturel de l'apparence historique l'emporte sur la 'signification'. Son apparence est plus difficile à dissiper. Elle est par ailleurs la plus récente " (B 462).

15. W. Benjamin, Das Passagen-Werk, Gesammelte Schriften, V, Suhrkamp, Frankfurt/Main, 1982, p. 60.

16. Cf. W. Hamacher, Über einige Unterschiede zwischen der Geschichte literarischer und der Geschichte phänomenaler Ereignisse, in M. Rauch et al. (Hrsg.), Texte zur Theorie und Didaktik der Literaturgeschichte, Stuttgart, Ph. Reclam jun., 2012, pp. 163-182.

17. W. Benjamin, Sur le concept d'histoire, in EEuvres III, Paris, Gallimard, 2000, p. 440 . Cette rencontre est une rencontre avec «ce qui est mort une fois pour toutes » : "L'intérêt que porte l'historien matérialiste au passé est toujours, en partie, un ardent intérêt pour ce qu'il a de révolu, pour ce qui en lui a cessé d'être et qui est mort une fois pour toutes » (B 545).

18. Ch. Péguy, Clio, Paris, Gallimard, 2002, p. 230.

19. W. Benjamin, Über den Begriff der Geschichte, Kritische Gesamtausgabe 19, Berlin, Suhrkamp, 2010 , p. 67.

20. Ibid.

21. W. Benjamin, Le narrateur, in CEuvres, Paris, Denoël-Gonthier, 1983.

\section{INDEX}

Mots-clés : Baudelaire (Charles), Benjamin (Walter), allégorie 